\title{
Orthogonal Cross Cylinder Using Segmentation Based Environment Modeling
}

\author{
Seung Taek Ryoo, Kyung Hyun Yoon \\ Department of Image Engineering \\ Graduate School of Advanced Imaging Science, Multimedia and Film \\ ChungAng University, Seoul, Korea \\ \{bluelancer, khyoon\}@cglab.cse.cau.ac.kr \\ http://cglab.cse.cau.ac.kr
}

\begin{abstract}
Orthogonal Cross Cylinder (OCC) mapping and segmentation based modeling methods have been implemented for constructing the image-based navigation system in this paper. The OCC mapping method eliminates the singularity effect caused in the environment maps and shows an almost even amount of area for the environment occupied by a single texel. A full-view image from a fixed point-of-view can be obtained with OCC mapping although it becomes difficult to express another image when the point-of-view has been changed. The OCC map is segmented according to the objects that form the environment and the depth value is set by the characteristics of the classified objects for the segmentation-based modeling. This method can easily be implemented on an environment map and makes the environment modeling easier through extracting the depth value by the image segmentation.
\end{abstract}

\section{Introduction}

With the development of image-based representation technology, it has become possible to navigate through the surrounding environment without actually modeling and constructing the virtual environment just by acquiring the image of the area to navigate. Photogrammetric modeling, image based modeling using corresponding points, interpolation from dense samples, cylinder based rendering, and the vanishing point based modeling method are generally used to construct an image-based navigation system [1]. With previous methods, it is difficult to acquire an image with a full-view when navigating through the environment. Also, the earlier methods are difficult to apply on environment maps. A new environment mapping method, and an environment modeling method through image segmentation are introduced in this paper to resolve such a problem.

The Orthogonal Cross Cylinder (OCC) is the object expressed by the intersection area that occurs when a cylinder is orthogonal with another (Fig. 1). The OCC mapping method eliminates the singularity effect caused in the environment maps and displays an almost even amount of area for the environment occupied by a single texel. The full-view image can be obtained through such a mapping method but it is difficult to express the image when the point of view

P.M.A. Sloot et al. (Eds.): ICCS 2002, LNCS 2330, pp. 141- 50, 2002.

(C) Springer-Verlag Berlin Heidelberg 2002 

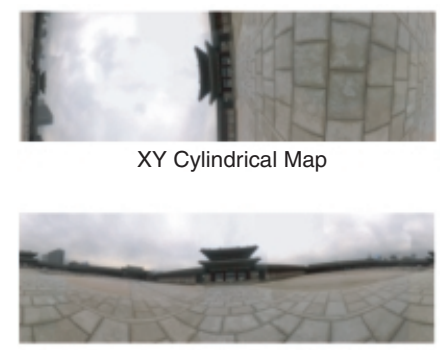

XZ Cylindrical Map

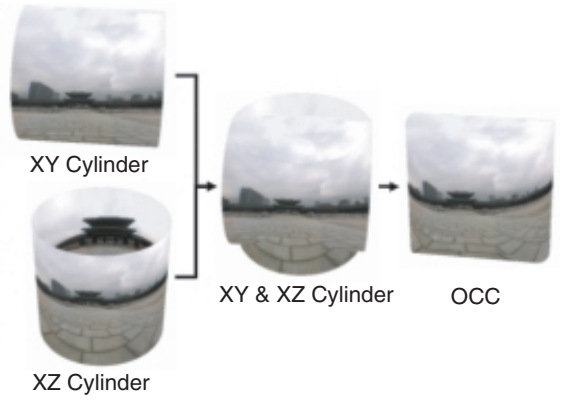

Fig. 1. Orthogonal Cross Cylinder

has been moved. The environment model must be composed using the depth value from the environment map to acquire the image from the changed point of view. The OCC map is divided by the object that forms the environment, and the depth value is set by the characteristics of the object in the segmentation based modeling method, which is used for this purpose. This method is easily applied on the environment map and can easily be used for the environment modeling by extracting the depth value through image segmentation. It becomes possible to develop an environment navigation system with a full-view through these methods.

\section{Related Work}

Environment mapping is a method of adding realism to a scene by using special texture indexing methods. This method refers to the process of reflecting a surrounding environment within a shiny object and it was originally introduced as a cheaper alternative to ray tracing [2]. In image-based rendering, the virtual viewer replaces the shiny object, and that part of the map intercepted by the viewer's field of vision is presented to him as a two-dimensional projection. Environment mapping is essentially the process of pre-computing a texture map and then sampling texels from this texture during the rendering of a model. The texture map is a projection of $3 \mathrm{D}$ space to $2 \mathrm{D}$ space. The methods used for the projection are sphere mapping, latitude mapping, cube mapping, cylindrical mapping, and paraboloid mapping [3].

Blinn/Newell Latitude Mapping used a latitude-longitude map indexed by the reflected ray [2]. The texture map's U coordinate represents longitude(from 0 to 360 degrees) and the $\mathrm{V}$ coordinate represents latitude(from -90 to 90 degrees). A latitude-longitude map's distortion will become apparent if the environment map is applied to planar objects with consistent neighborhood relationships such as a mirror. An object with curved surfaces will alleviate the distortion inherent in the environment map image. Furthermore, there is a singularity in the mapping at the pole. Sphere mapping is a type of environment mapping in which the irradiance image is equivalent to that which would be seen in a 
perfectly reflective hemisphere when viewed using an orthographic projection. Sphere mapping uses a single texture image. This means that look-ups are fast and the environment is represented compactly. But, there is a singularity in the mapping at the outer edge and it does not use all the texture in the texture map. There is a waste of texture memory. Cube mapping was invented by Ned Greene in 1986 [4]. The cube map stores the environment as six textures. Each texture represents the view out of one face of the cube as seen from the center of the cube. A cube map is easier to generate than a sphere map and results in less distortion than the other mappings. But, cube mapping requires indexing through several textures in order to compose the final image. Also, discontinuity is introduced near the edges of the map and this results in seams occurring along the object. For cylindrical mapping, a cylinder can be easily unrolled into a simple planar map. The surface is without boundaries in the azimuth direction. One shortcoming of a projection on a finite cylindrical surface is the boundary conditions introduced at the top and bottom [5]. Dual paraboloid environment mapping was invented in 1998 by Heidrich and Seidel [6]. Dualparaboloid mapping requires two textures to store the environment, one texture for the front environment and another texture for the back environment. The underlying geometry of the mapping is based on a parabola, which has the desirable property of containing a single point of focus for incoming rays. A dualparaboloid map compared to the sphere map approach is better sampling of the texture environment with the elimination of sphere mapping's sparkle artifacts. But, constructing the dual-paraboloid map requires warping two textures instead of just one and also requires two rendering passes or the use of multi-texturing. Also, almost $25 \%$ of the pixels are not used, and edge pixels between maps need to perform special blending in order to reduce artifacts from using two maps. In this paper, we propose the use of OCC mapping, which is a new type of mapping that eliminate the singularity problem occurring at the pole of the environment map and the problem of the size of the area (solid angle) subtended by a texel environment map of various forms. The properties of OCC mapping are presented in chapter 3 .

Environment modeling using environment information is required to freely navigate through the environment. Photogrammetric modeling, a modeling using the disparity acquired through a matching algorithm, and modeling using the vanishing point method are a couple of modeling methods [1]. A 3D model can be constructed through the method using the disparity, but it requires a high cost for searching the corresponding point from the image. The vanishing point based modeling method only constructs a rough model, which makes it difficult to express the details of the environment. With the photogrammetric modeling method, it is difficult to match the object and the primitive due to the deformation of the image when applied on the environment map. The segmentation based modeling method is used in this study to solve such a problem. This method can easily be applied on environment maps and can be used to model the environment easily. The segmentation based modeling method is discussed in detail in chapter 4 . 


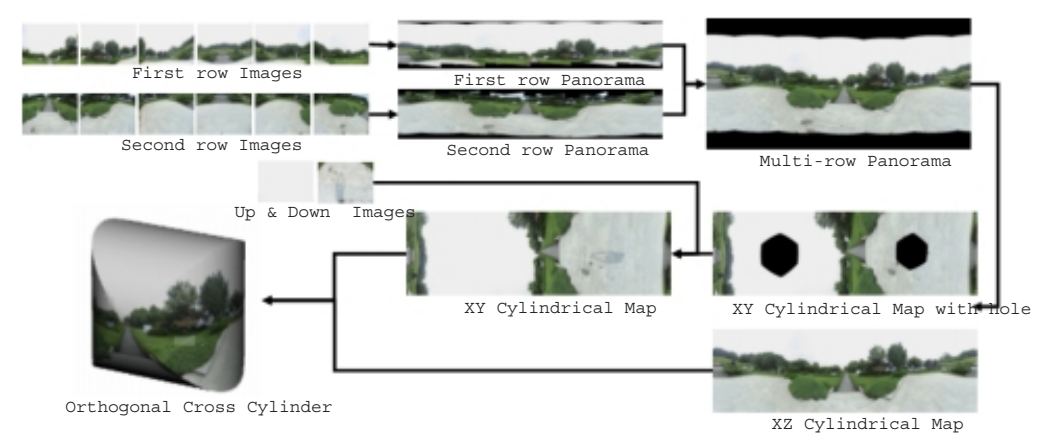

Fig. 2. Creation of OCC map from real scene

\section{Orthogonal Cross Cylinder}

OCC has been developed for evenly sampling the environment map by the pixels on the screen and its effective storage while maintaining the characters of the cylindrical map. It is an object created by intersecting the XZ cylinder of Y-axis and the XY cylinder of the Z-axis (Fig. 1).

The process of expressing the surrounding environment using OCC mapping consists of creating an XZ and XY cylindrical map, reproducing the OCC map through eliminating redundancy, and rendering through OCC map sampling. The environment map is created by using a virtual line camera in the environment which creates three dimensions when created from computer-generated images and by using the stitching algorithm when created from real-world images. The XZ and XY cylindrical maps created through these methods contain ineffective samples that overlap. Therefore, a step for eliminating such redundant samples is required in the process of constructing a more effective OCC map. The OCC map is composed in a way to be compatible with other environment maps. An image can be created in any direction through rendering based on texture mapping and ray casting using the OCC map created.

\subsection{Creation of OCC Map}

Figure 2 is a flow diagram of the process to construct an OCC from a real scene. An image is acquired by pitching up (using a tripod) the camera with a semi fisheye lens attached, by 30 degrees off the horizontal plane and then taking the six images by rotating anti-clockwise around the Y-axis at 30 degree intervals. The camera is then pitched down by 30 degrees off the horizontal plane and rotated around the $\mathrm{Y}$-axis to acquire the six images in 30 degree intervals. Finally, the bottom and the top of the scene, after the tripod has been removed, are captured as an image. The images acquired from the first rotation are stitched together as a cylindrical map using the panorama tool. The images acquired from the next rotation are put together to form the second cylindrical map. A panoramic image with a wide angle can be obtained by stitching two different cylindrical 


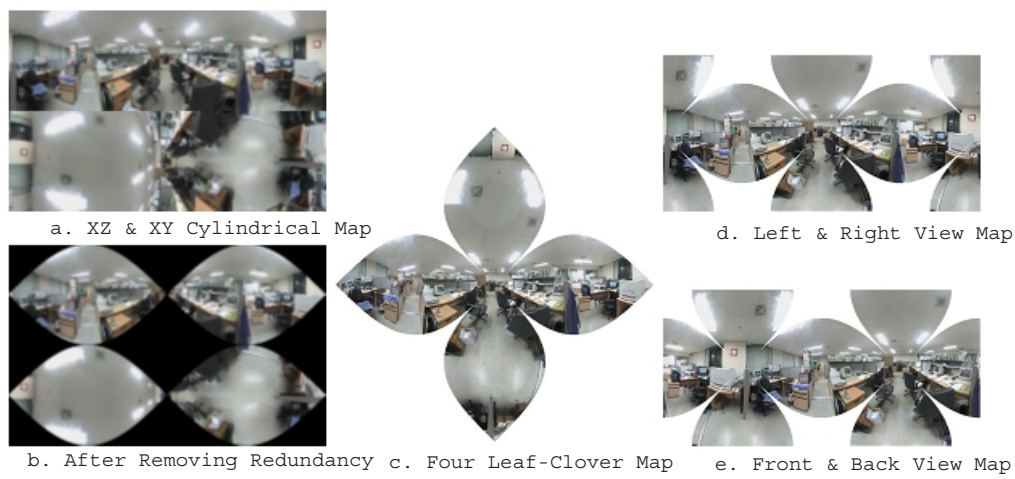

Fig. 3. Various environment maps using OCC

maps together in the same way. However, the panoramic image obtained through this method cannot include its entire surrounding environment due to the lack of information on its surrounding environment around each pole. The panoramic images are transformed into an XZ and an XY cylindrical map to resolve this problem. The transformed XZ cylindrical map produces a perfect solution by acquiring all the necessary information on its surrounding environment, whereas the solution from the XY cylindrical map would have holes due to the lack of information on the top and bottom of the image. These holes can be filled by using the image of the top and bottom acquired in the first step of the process and an image-editing program.

An OCC can be constructed using the XZ and XY cylindrical maps created through this process. However, the $\mathrm{XZ}$ and $\mathrm{XY}$ cylindrical maps used to create the OCC are not suitable to be used as OCC maps because they contain redundant environment data. This problem is discussed in the following chapter.

\subsection{The Shape of OCC Map}

The easiest way of mapping the OCC onto a $2 \mathrm{D}$ screen coordinate is to use the XZ and XY cylindrical map (Fig. 3-a). However, since the two cylindrical maps contain redundant environment data, it is necessary to discard such redundancy beforehand. The following equation can be obtained by finding the intersection area of the two cylinders using the radius $(r)$ of the cylinder and the coordinate of the cylinder $(\theta, v)$.

$$
v=r \pm r \sin \frac{\theta}{r}
$$

The redundant data of the two cylinders can be discarded using equation 1 (Fig. 3-b). But, this cylindrical map is ineffective by having $35 \%$ of its storage space left as empty space. The XZ and XY cylindrical map is similar in its shape to a four-leaf clover (Fig. 3-c). Each end of the leaf of the clover meets at a single point. A map that maps the right side and the left side of the OCC (Fig. 3-d) 


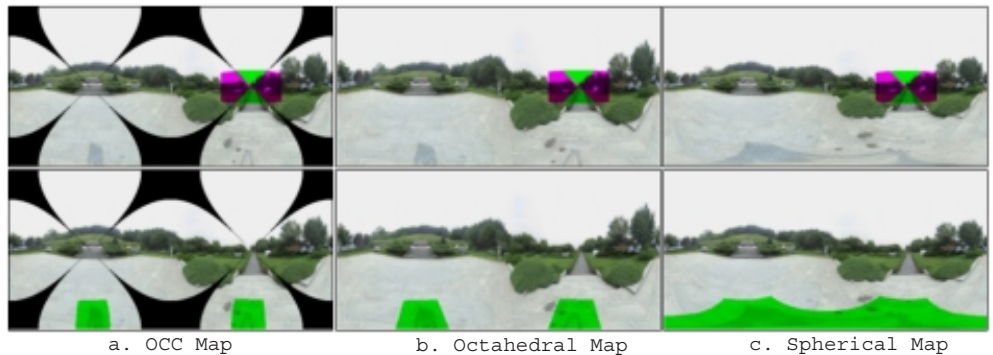

Fig. 4. Comparing the area that the screen pixels occupy

and a map that maps the front and the backside of the OCC (Fig. 3-e) can be constructed through this characteristic of the OCC map. The space for storing the samples needed to construct an OCC can be reduced by $25 \%$ and wasted space can be reduced by $19 \%$ through this method. The front and the backside of the map can be used through warping for even more effective use of space (Fig. 4). The octahedral map is formed through warping the pixels towards the straight line to fill the space left empty in the front and the back-view map.

\subsection{Comparing Sampling Pattern}

In this study, the accuracy of each map has been evaluated by comparing the texture area corresponding to a certain solid angle and the solid angle corresponding to each texel. The size of the area that the screen pixel occupies is more even at the pole and near the equator for the OCC map and the octahedral map, than the spherical map, as shown in Figure 4.

Also, the solid angle for each texel has been calculated to measure the effectiveness of the sampling on each map. The solid angle of the texel for the cube map, cylindrical map, spherical map, OCC map, and the octahedral map have each been measured in this study. The solid angle is expressed as the area that the ray has been projected onto the sphere for each texel

Table 1. Comparing solid angle

\begin{tabular}{|c|c|c|c|c|c|c|c|}
\hline & Sample \# & Total & Min. & Max. & Avg. & Dev. & Area \\
\hline Cube & 239756 & 12.573205 & 0.000019 & 0.000100 & 0.000052 & 0.000020 & $100 \%$ \\
\hline Cylinder & 125600 & 8.888219 & 0.000036 & 0.000100 & 0.000071 & 0.000021 & $71 \%$ \\
\hline Sphere & 197192 & 12.566737 & 0.000001 & 0.000100 & 0.000064 & 0.000031 & $100 \%$ \\
\hline OCC & 159764 & 12.559790 & 0.000036 & 0.000100 & 0.000079 & 0.000019 & $100 \%$ \\
\hline Octahedron & 197192 & 12.566234 & 0.000023 & 0.000100 & 0.000064 & 0.000020 & $100 \%$ \\
\hline
\end{tabular}

Table 1 shows the minimum and maximum value, average value, and the deviation of the solid angle on each map. If the average value is small, the area 


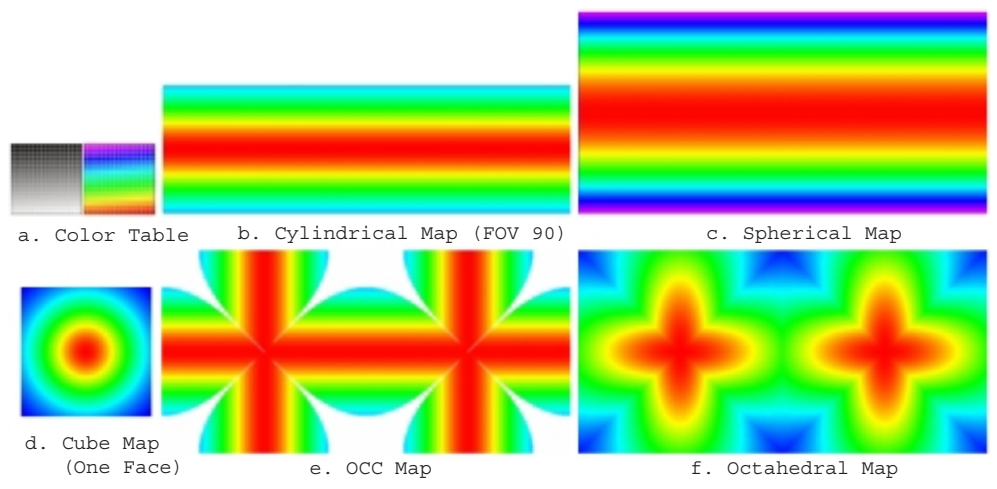

Fig. 5. Comparing solid angle per single texel

of the environment that the texel represents is of a small size and vice versa. The deviation value represents the change in the value of the solid angle. The change in the value of the angle becomes more even as the deviation value becomes smaller. Therefore, an effectively sampled map has a large average value and a small distribution value. The cube map has the smallest average value of all the maps, but has a small deviation value, which causes little change in the value of the solid angle of a texel. The cylindrical map shows a good sampling pattern with a large average value and a small distribution value, but has a problem of not being able to cover all the area. The spherical map has a sampling pattern that shows a rapid decrease of the value for the solid angle at the pole, and a large difference in the value between the minimum and maximum value of the solid angle. The OCC map has the best sampling pattern of them all, with the largest average value and the smallest distribution value. A spectrum color table has been used to visually express the difference between the solid angle of each texel for the various maps, instead of using the grey shade color table, in Figure 5 -a. Figure 5 shows that the OCC map provides the best sampling pattern, which expresses the surrounding environment most effectively.

\section{Segmentation Based Modeling}

The OCC method, explained above, is used to navigate its surrounding environment from a fixed point of view. However, a model that uses the environment map is required to freely navigate through the virtual environment. The depth value must be extracted from the OCC map to construct such a model. The segmentation based modeling method is used for this purpose in this study.

\subsection{Image Segmentation}

An indoor virtual environment consists of a floor and ceiling and the outdoor of ground and sky. From such features, the environment map is divided into a floor 
(ground), ceiling (sky), and the surrounding objects. A method for image segmentation is required to divide the environment map into these characteristics. The image segmentation method can be divided into a region-based method and an edge-based method [7][8]. Region based methods take the basic approach of dividing the image into regions and classifying pixels as inside, outside, or on the boundary of a structure based its location and the surrounding $2 \mathrm{D}$ regions. On the other hand, the edge-based approach classifies pixels using a numerical test for a property such as image gradient or curvature. The environment map can be segmented by the characteristics of the object through these methods.

\subsection{Depth Calculation}

The depth can easily be acquired from the segmented environment map using the following conditions:

- The floor (ground) and the ceiling consist of a plane that is parallel to the $\mathrm{XZ}$ plane in the real-world coordinate.

- The sky consists of a semi-sphere with an infinite radius.

- The surrounding objects are perpendicular to the ground.
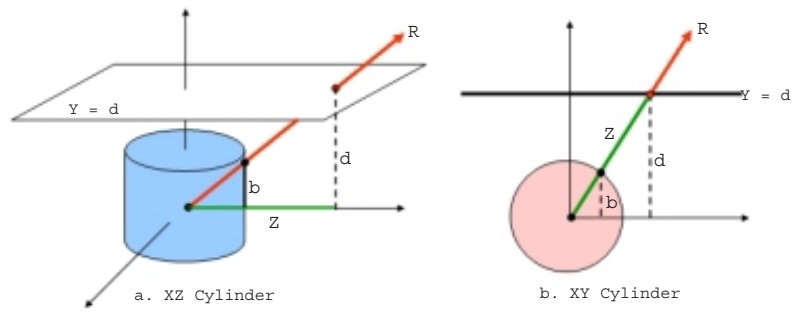

Fig. 6. Depth calculation using the relationship between ceiling (floor) and OCC

Figure 6 shows the process of extracting the depth value of the ceiling. The depth can be obtained by testing for the intersection point between the ceiling that is parallel to the XZ plane, and the ray that crosses the actual 3D coordinate mapped onto the pixel on the environment map. The relationship between the XZ cylinder and the ray (Fig.6-a), and between the XY cylinder and the ray (Fig. 6-b) must be considered to get the depth value from the OCC map. The following equation can be obtained by calculating the radius $(r)$ of the cylinder, height $(d)$ of the XZ plane, and the y coordinate value $(b)$ of a point on the cylinder with the triangle equation.

$$
Z(\text { Depth })=r \times \frac{d}{b}
$$

The depth of the floor and the ceiling of the segmented environment map can be extracted using Equation 2. Also, because the surrounding objects are 
perpendicular to the ground, the depth image can be obtained by setting the depth value of the floor on the objects surrounding the floor. Figure 7 shows the process of environment mapping from OCC map and environment modeling from depth image. Figure 8 compares three frames that have been rendered using the OCC model and the Environment model using depth image. The image above is the OCC model image, and the image below is the environment model expressed by adding a depth image to the OCC model. It should be evident that the OCC model with depth, expresses the environment well by observing the occlusion in the wall in the middle.

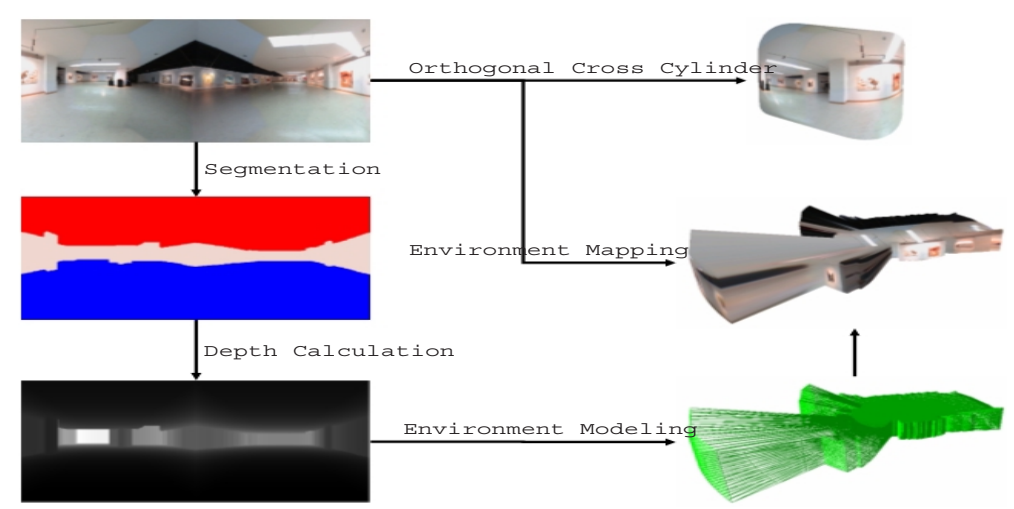

Fig. 7. Environment mapping from OCC map and Environment modeling from depth image

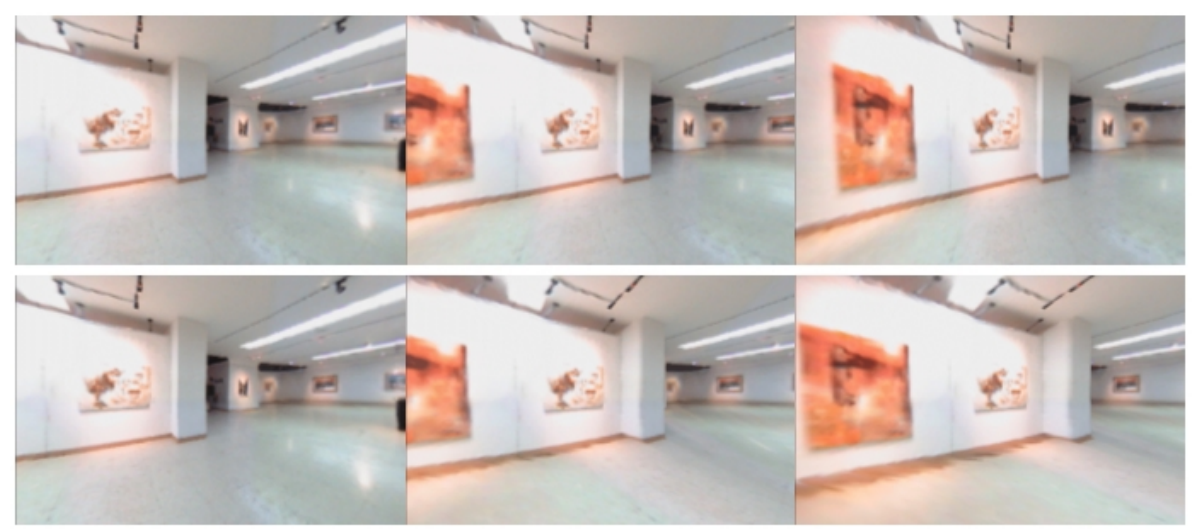

Fig. 8. Comparing of OCC and segmentation based model 


\section{Conclusion \& Future Works}

The OCC mapping method, a new method used for effective environment mapping and a segmentation-based environment modeling method for freely navigating through the environments are introduced in this study. The OCC mapping method eliminates the singularity effect caused in the environment maps and shows an almost even amount of area for the environment occupied by a single texel. The OCC mapping method is suitable for application on environment navigation systems due to its effective storage of the environment and faster sampling time.

The segmentation-based environment modeling method is easy to implement on the environment map and can be used for environment modeling by extracting the depth value by the segmentation of the environment map. However, an environment model that is constructed using a single OCC map has the problem of a blurring effect caused by the fixed resolution, and the stretching effect of the 3D model caused when information that does not exist on the environment map occurs due to the occlusion. A study on voronoi-diagram based environment modeling and environment mapping using multiple OCC maps is underway to solve these problems. Also, the surrounding objects are set to be perpendicular to the floor upon the image segmentation to set the depth as the depth of the neighboring floor. It is difficult to express the surrounding objects in detail with these modeling methods. An environment modeling method that subdivides the surrounding objects is required to express the objects in detail.

\section{Acknowledgement}

This work was partially supported by the National Research Laboratory program of the Korean Ministry of Science and Technology (No. 2000-N-NL-01-C-285)

\section{References}

1. Bregler, C., Cohen, M. F., Debevec, P., McMillan L., Sillion, F. X., Szeliski, R.: Image-based Modeling, Rendering, and Lighting. Siggraph 2000 Course 35, (2000)

2. Blinn J., Newell M.: Texture and reflection in computer generated images. Communications of the ACM, (1976) 19:456-547

3. Blythe, D., Grantham, B., McReynolds, T., Nelson, S. R.: Advanced Graphics Programming Techniques Using OpenGL. Siggraph '99 Course 29, (1999)

4. Greene, N.: Environment Mapping and Other Applications of World Projections. Computer Graphics and Applications, (1986) 6(11):21-29

5. McMillan, L., Bishop, G.: Plenoptic modeling : An image-based rendering system. Siggraph '95, (1995) 39-46

6. Heidrich, W., Seidel, H. -P.: View independent Environment Maps. Eurographics/ACM Siggraph Workshop on Graphics Hardware '98, (1998) 39-46

7. Imielinska, C., Laino-Pepper, L.: Technical Challenges of 3D Visualization of Large Color Data Sets. The Second Visible Human Project Conference Proceedings, (1998)

8. Mortensen, E. N., Reese, L. J., Barrett, W. A.: Intelligent Selection Tools. IEEE Conference on Computer Vision and Pattern Recognition '00, (2000) 776-777 